\title{
A Robust Mammary Organoid System to Model Lactation and Involution-like Processes
}

Elsa Charifou' ${ }^{1}$, Jakub Sumbal ${ }^{1,2}$, Zuzana Koledova², Han Li $^{1}$ and Aurélie Chiche ${ }^{1, *}$

${ }^{1}$ Cellular Plasticity \& Disease Modeling - Department of Developmental \& Stem Cell Biology, CNRS UMR3738 - Institut Pasteur, 25 rue du Dr Roux, Paris 75015, France; ${ }^{2}$ Department of Histology and Embryology, Faculty of Medicine, Masaryk University, Kamenice 3, Brno 625 00, Czech Republic *For correspondence: aurelie.chiche@pasteur.fr

[Abstract] The mammary gland is a highly dynamic tissue that changes throughout reproductive life, including growth during puberty and repetitive cycles of pregnancy and involution. Mammary gland tumors represent the most common cancer diagnosed in women worldwide. Studying the regulatory mechanisms of mammary gland development is essential for understanding how dysregulation can lead to breast cancer initiation and progression. Three-dimensional (3D) mammary organoids offer many exciting possibilities for the study of tissue development and breast cancer. In the present protocol derived from Sumbal et al., we describe a straightforward 3D organoid system for the study of lactation and involution ex vivo. We use primary and passaged mouse mammary organoids stimulated with fibroblast growth factor 2 (FGF2) and prolactin to model the three cycles of mouse mammary gland lactation and involution processes. This 3D organoid model represents a valuable tool to study late postnatal mammary gland development and breast cancer, in particular postpartum-associated breast cancer.

\section{Graphic abstract:}

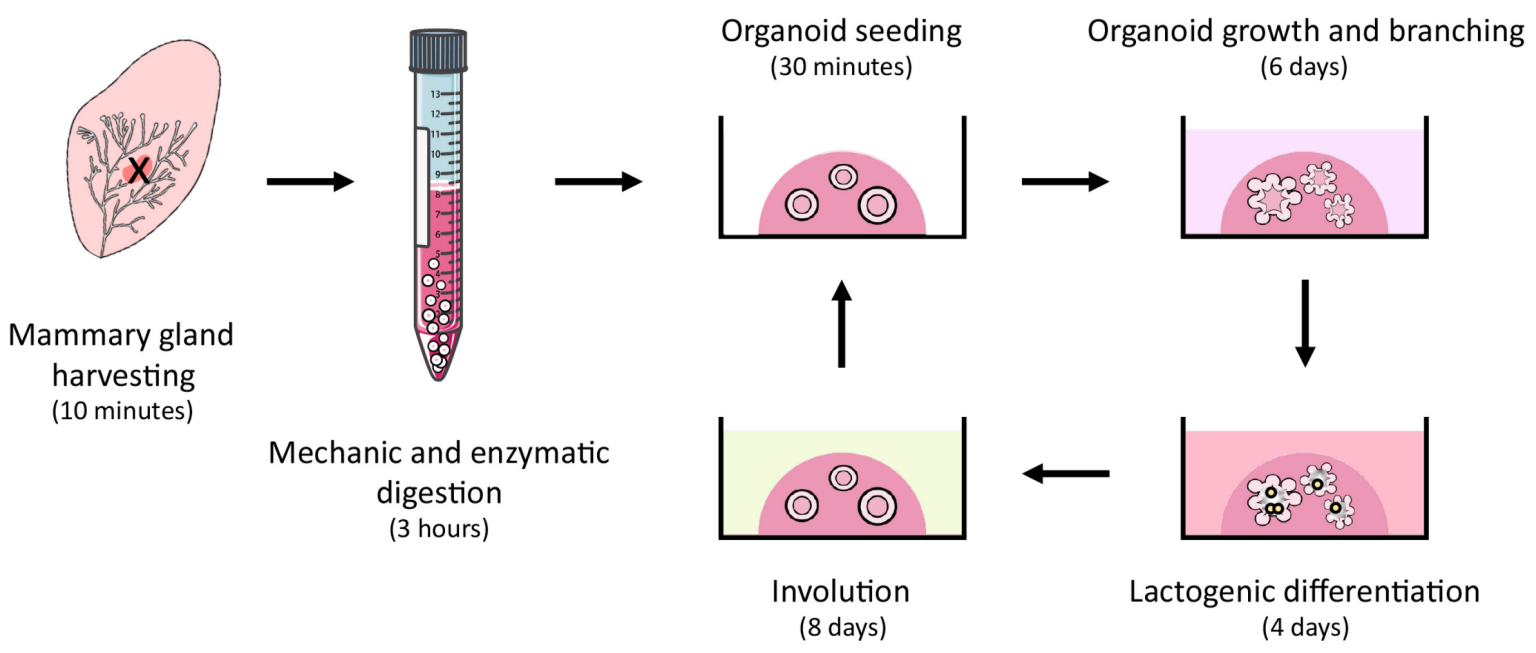

\section{Mammary gland organoid isolation and culture procedures}

Keywords: Mouse, Mammary gland, 3D organoid, Ex vivo, Lactation, Involution 
[Background] The primary function of the mammary gland is to provide nutrition to newborns via milk production. The development of the mammary gland is a highly dynamic process that occurs mainly after birth and is regulated by several factors including hormones and growth factors (Brisken and Rajaram, 2006; Sternlicht, 2006). During puberty, hormones and growth factors regulate ductal morphogenesis from a rudimentary embryonic ductal tree (Brisken and O'Malley, 2010). During each pregnancy, the mammary gland begins a new morphogenetic step initiated by hormonal stimulation, which is characterized by massive proliferation for epithelial expansion and alveolar development accompanied by adipocyte regression (Brisken and O'Malley, 2010). Importantly, prolactin signaling plays a crucial role in the terminal differentiation of luminal cells to enable milk production (Ormandy et al., 1997). At the end of lactation after weaning of the progeny, the mammary gland enters the involution stage characterized by programmed cell death, tissue remodeling, and redifferentiation of adipocytes (Hughes and Watson, 2012; Macias and Hinck, 2012; Zwick et al., 2018; Jena et al., 2019).

Histologically, the mammary gland is composed of a bilayered epithelium consisting of an inner layer of luminal cells (keratin $8^{+}$) and an outer layer of contractile basal cells (keratin $5^{+}$). Luminal cells are responsible for milk production during lactation, while basal cells aid milk ejection. The epithelium is surrounded by a stromal fat pad that comprises fibroblasts, nerves, vasculature, lymphatics, immune cells, adipocytes, and extracellular matrix (ECM) (Richert et al., 2000).

Over the past decade, organoids of various tissues, such as stomach, colon, lung, and pancreas, have been developed (Huch and Koo, 2015), offering many exciting possibilities for the study of tissue development and disease. The organoid system is a powerful tool that combines the advantages of a 2D culture (easy manipulation, precise control of cell composition and microenvironment, live imaging) with the opportunity to study complex cell-cell and cell-ECM interactions in a more controlled ex vivo manner (Huch and Koo, 2015; Shamir and Ewald, 2015; Koledova, 2017; Artegiani and Clevers, 2018).

Several models have been developed to study the mechanisms of mammary branching morphogenesis in primary mammary epithelium using different protocols (Ewald et al., 2008; Huebner et al., 2016; Neumann et al., 2018), cell lines (Xian et al., 2005), sorted cells (Jamieson et al., 2017; Linnemann et al., 2015), or induced pluripotent stem cells (Qu et al., 2017). However, an organoid system modeling key aspects of the late postnatal developmental stages of the mammary gland has remained challenging to establish.

Previously, there have been several attempts to model lactation in 3D culture: spheroids of a breast adenoma cell line were used to study copper secretion into milk (Freestone et al., 2014); organoids of primary epithelium were shown to produce milk following the administration of a lactogenic stimulus (Mroue et al., 2015; Jamieson et al., 2017); and co-culture of breast epithelium and pre-adipocyte cell lines was shown to initiate an involution-like process (Campbell et al., 2014). However, in-depth characterization of milk production and involution or the proper bilayered architecture of mammary epithelium remained to be carried out.

Recently, we developed a model of lactation and involution of mammary epithelium based on organoids of primary mammary gland tissue cultured in 3D Matrige ${ }^{\circledR}$ (Sumbal et al., 2020b). Under lactogenic stimuli, primary organoids maintain long-term milk production, retain the contractile 
myoepithelial layer, and enter involution following hormone withdrawal. Moreover, after involution, the organoids remain hormonally sensitive and are able to enter another round of lactation (Sumbal et al., 2020b). Here, we present a methodological guideline to establish the primary mammary organoid-based ex vivo model of lactation and involution, with detailed procedures for obtaining tissue, isolating organoids, establishing and maintaining 3D culture, and preparing organoid samples for subsequent RNA or protein expression analysis or histological examination. This model can be used for studies on lactation biology, mammary stem cell plasticity, regulatory mechanisms of mammary epithelial cell differentiation and death, or other interesting biological phenomena. We believe that this model will initiate the further development of organoid technology, including creative applications in biotechnology and regenerative medicine (Sumbal et al., 2020a).

\section{Materials and Reagents}

1. 100-mm tissue culture Petri dish (e.g., Corning, catalog number: 353003 )

2. $0.2-\mu \mathrm{m}$ filters and $50 \mathrm{ml}$ syringes (e.g., GVS, catalog number: FJ25ASCCA002DL01)

3. No. 22 disposable scalpel blades (e.g., Swann-Morton, catalog number: 0508)

4. 50-ml tubes (e.g., Corning, catalog number: 352070)

5. 15-ml tubes (e.g., Corning, catalog number: 352096)

6. 10-ml disposable plastic pipettes (e.g., Corning, catalog number: 357551)

7. 25-ml disposable plastic pipettes (e.g., Corning, catalog number: 357535)

8. 24-well tissue culture plates (e.g., Corning, catalog number: 353047)

9. $30 \mathrm{G}$ insulin syringes (e.g., BD Microfine, catalog number: 324826 )

10. Plastic histology molds (e.g., Thermo Scientific, catalog number: 1830)

11. Plastic embedding cassettes (e.g., Simport, catalog number: M492-2)

12. Histology tissue molds (e.g., Simport, catalog number: M474-3)

13. Microscope slides for histology (e.g., Thermo Scientific, catalog number: J1800AMNZ)

14. Mice: virgin females, 7-10 weeks old, inbred strain C57BL/6J (e.g., The Jackson Laboratory, catalog number: 000664)

15. Ethanol (EtOH), 70\%, 95\%, and 100\% (e.g., VWR, catalog number: 83813)

16. Phosphate-buffered saline (PBS) (e.g., Sigma-Aldrich, catalog number: D1408)

17. Dulbecco's modified Eagle medium (DMEM)/F12 (e.g., Gibco, catalog number: 21331-020)

18. Bovine serum albumin (BSA) (e.g., Sigma-Aldrich, catalog number: A3608)

19. Fetal bovine serum (FBS) (e.g., Sigma-Aldrich, catalog number: F0804)

20. Collagenase A (e.g., Roche, catalog number:11088793001)

21. Trypsin (e.g., Dutcher Dominique, catalog number: P10-022100)

22. Insulin (e.g., Sigma-Aldrich, catalog number: I6634-100MG)

23. Gentamicin (e.g., Sigma-Aldrich, catalog number: G1397)

24. Glutamine (e.g., Gibco, catalog number: 35050-061)

25. DNase I (e.g., Sigma-Aldrich, catalog number: D4527-40KU) 
26. Dispase II (e.g., Roche, catalog number: 1375 2000)

27. Growth factor-reduced Matrige ${ }^{\circledR}$ (e.g., Corning, catalog number: 354230$)$

28. Insulin-transferrin-selenium (ITS) (e.g., Gibco, catalog number: 41400-045)

29. Penicillin/Streptomycin (e.g., Gibco, catalog number: 15140-122)

30. FGF2 (e.g., Gibco, catalog number: PM60034)

31. Prolactin (e.g., Sigma-Aldrich, catalog number: SRP4688)

32. Hydrocortisone (e.g., Sigma-Aldrich, catalog number: S H6909)

33. Oxytocin (e.g., Sigma-Aldrich, catalog number: 03251)

34. RNeasy Micro Kit (e.g., Qiagen, catalog number: 74004)

35. $\beta$-Mercaptoethanol (e.g., Sigma-Aldrich, catalog number: M6250)

36. Phosphatase inhibitor cocktail II (e.g., Millipore, catalog number: 524625)

37. RIPA buffer (e.g., Sigma-Aldrich, catalog number: R0278)

38. Protease inhibitor cocktail I (e.g., Sigma-Aldrich, catalog number: 539131)

39. Pierce Coomassie (Bradford) Protein Assay Kit (e.g., Thermo Scientific, catalog number: 23200)

40. Paraformaldehyde (PFA), 32\% (e.g., Electron Microscopy Sciences, catalog number: 15714)

41. Low gelling temperature agarose (e.g., Sigma-Aldrich, catalog number: A9414)

42. Xylene (e.g., Sigma-Aldrich, catalog number: 534056)

43. Paraffin (e.g., Sigma-Aldrich, catalog number: 1071642504)

44. Dissociation solution (see Recipes)

45. BSA solution (see Recipes)

46. Basal organoid medium (BOM) (see Recipes)

47. Morphogenesis medium (see Recipes)

48. Lactation medium (see Recipes)

49. 4\% PFA (see Recipes)

50. RNA lysis buffer (see Recipes)

\section{Equipment}

1. Surgical tools

Forceps (e.g., Phymep, catalog numbers: $11050-10$ and 11051-10)

Scissors (e.g., Phymep, catalog number: 14088-10)

2. Dissection board (e.g., Thermo Scientific, catalog number: 36-119)

3. P1000 pipette

4. Laminar flow hood

5. Fridge $4^{\circ} \mathrm{C}$ (e.g., Liebherr, catalog number: 7083 001-01)

6. Freezer $-80^{\circ} \mathrm{C}$ (e.g., Thermo Scientific, catalog number: $88400 \mathrm{~V}$ )

7. Liquid nitrogen tank (e.g., Air Liquide Espace 151, catalog number: 2433867)

8. Shaking incubator at $37^{\circ} \mathrm{C}$ (e.g., Infors HT Multitron)

9. Centrifuge (e.g., Thermo Scientific, model: Sorvall ST40) 
10. Incubator for cell culture, $37^{\circ} \mathrm{C}, 5 \% \mathrm{CO}_{2}$ (e.g., Thermo Scientific, model: HERAcell 150i)

11. Heating plate at $37^{\circ} \mathrm{C}$ (e.g., Techne DRI-Block DB-2A)

12. Microscope and camera (e.g., Olympus model: CKX41)

13. NanoDrop ${ }^{\mathrm{TM}}$ (e.g., Implen Nanophotomoter NP80)

14. Sonicator (e.g., Diagenode Bioruptor Pico)

15. Incubator at $65^{\circ} \mathrm{C}$ (e.g., Memmert Incubator I)

16. Embedding workstation (e.g., Leica EG1150C)

\section{Procedure}

A. Isolation of mammary primary organoids

1. Dissection of a virgin mouse to harvest mammary glands (see Video 1).

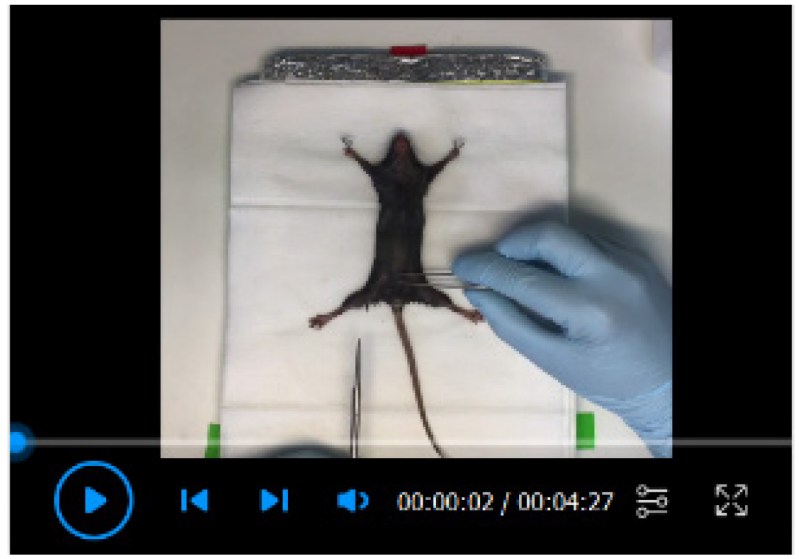

Video 1. Mammary gland harvesting. This video was made at Pasteur Institute. according to guidelines from the regulations of Institut Pasteur Animal Care Committees (CETEA). on Animal Care and approved by the French legislation in compliance with European Communities Council Directives (A 75-15-01-3).

a. Euthanize the donor mouse using an ethically approved method (e.g., cervical dislocation) and immediately proceed to mammary gland collection.

Notes:

i. Cervical dislocation is a common method for animal euthanasia and provides a fast and painless death. With this method, cell/tissue survival in culture is not altered if collected immediately.

ii. In the case of processing multiple mice, euthanize one animal and collect the glands immediately, then proceed to the next animal.

b. Sanitize the ventral side of the animal by spraying $70 \% \mathrm{EtOH}$ on the skin.

Note: After disinfection, work inside a laminar flow hood to maintain aseptic conditions. Application of aseptic work procedures, together with the presence of antimycotic and 
antibiotic supplements (gentamicin in digestion solution; penicillin and streptomycin in culture medium) will prevent the occurrence of contamination.

c. Pin the mouse by its four paws to a dissection board, with the abdomen facing upward (see Figure 1A, pins 1-4).

d. Using forceps, tightly grasp the skin of the lower part of the abdomen at half the width (see Figure $1 \mathrm{~A}$, point $\mathrm{A}$ ).

e. Using surgical scissors, make the first incision in the skin at point $A$.

Note: Be careful to incise only the skin and not rupture the underlying peritoneum.

f. Continue to incise the skin cranially to the throat of the animal (see Figure $1 \mathrm{~A}$, from point $\mathrm{A}$ to point B).

g. From this median line, use forceps to grasp the skin and cut toward each of the four paws (see Figure $1 \mathrm{~A}$, incise to join the middle line to points $\mathrm{C}, \mathrm{D}, \mathrm{E}$ or $\mathrm{F}$, respectively).

h. Using forceps and a cotton swab, gently separate the skin from the peritoneum on one side of the animal. Attach the skin to the dissection board with three pins (see Figure 1B, pins 57).

i. Repeat step 8 on the other side of the animal (see Figure 1B, pins 8-10). The mammary glands are now exposed.

j. Identify the lymph node of the mammary gland \#4 (a small dense structure, round in shape; see Figure 1B, surrounded). Remove the lymph node from both glands using forceps and scissors and discard.

k. Proceed to the harvest of the mammary glands \#3 and \#4. Using curved forceps, grasp the mammary glands and gently separate them from the skin and other tissues with scissors. Note: Carefully separate the mammary glands \#3 (whitish and shiny) from the muscles (light brown ribbed structure) since this protocol does not prevent muscle contamination.

I. Place all the collected glands in the same sterile Petri dish containing cold PBS (approximately $3 \mathrm{ml}$, previously stored at $4^{\circ} \mathrm{C}$ ) for washing prior to tissue processing.

$\mathrm{m}$. Properly dispose of the animal corpse and continue with mechanical and enzymatic dissociation of the mammary glands.

2. Mechanical and enzymatic dissociation

Reminder: Work inside a laminar flow hood to maintain aseptic conditions.

a. Freshly prepare $10 \mathrm{ml}$ dissociation solution for the four glands collected from one mouse, pass through a $0.2-\mu \mathrm{m}$ filter, and pre-heat at $37^{\circ} \mathrm{C}$.

Note: Do not exceed the maximum $30 \mathrm{ml}$ dissociation solution in a 50-ml tube to ensure correct dissociation.

b. Transfer the freshly collected mammary glands to a new sterile Petri dish.

c. Use three scalpels to finely chop the mammary glands and obtain a homogeneous mince of $1-\mathrm{mm}^{3}$ mammary fragments (see Figure $1 \mathrm{C}$ ).

d. Transfer the mince to a 50-ml tube containing the pre-warmed dissociation solution.

e. Place the tube in a shaking incubator for $30 \mathrm{~min}$ at $37^{\circ} \mathrm{C}, 100 \mathrm{rpm}$. 
Notes:

i. All the following steps are performed at room temperature except incubation with dispase.

ii. From here on, pre-coat all the pipettes, tips, and tubes with $2.5 \%$ BSA solution. Prepare the BSA solution in a 50-ml tube and aspirate/remove from every consumable following coating; this will prevent stickiness and loss of organoids. The BSA solution can then be filtered, stored at $4^{\circ} \mathrm{C}$, and re-used.

f. After incubation, resuspend the dissociated mammary glands by performing ten up-anddown motions with a 10-ml pipette. Centrifuge for $10 \mathrm{~min}$ at $400 \times \mathrm{g}$.

g. After centrifugation, handle the $50-\mathrm{ml}$ tube carefully to prevent disturbance of the three separated layers (see Figure 1C). Keep the epithelial pellet intact and transfer the middle aqueous phase and the top fatty layer into a clean 15-ml tube.

h. Resuspend the epithelial pellet in $5 \mathrm{ml}$ DMEM/F12 and set it aside.

i. Focus on the fatty and aqueous solutions in the 15-ml tube: resuspend by performing ten up-and-down motions with a $10-\mathrm{ml}$ pipette. Centrifuge for $10 \mathrm{~min}$ at $400 \times \mathrm{g}$.

Note: This step allows recovery of epithelial fragments trapped in the fatty layer.

j. Again, handle the $15-\mathrm{ml}$ tube carefully to avoid disturbing the three separated layers. Discard the fatty and aqueous layers.

k. Take the $5 \mathrm{ml}$ resuspended pellet from the $50-\mathrm{ml}$ tube to resuspend the pellet in the $15-\mathrm{ml}$ tube.

I. Wash the 50-ml tube with $5 \mathrm{ml}$ DMEM/F12, pool with the suspension in the $15-\mathrm{ml}$ tube, and mix.

m. Centrifuge for $10 \mathrm{~min}$ at $400 \times \mathrm{g}$.

n. Discard the supernatant. Use $4 \mathrm{ml} D M E M / F 12$ to resuspend the pellet. Subsequently, add $80 \mu \mathrm{l} \mathrm{DNAse} \mathrm{I} \mathrm{at} 100 \mu \mathrm{g} / \mathrm{ml}$ and agitate for $5 \mathrm{~min}$ by hand or on an orbital shaker at $100 \mathrm{rpm}$.

o. Add $6 \mathrm{ml}$ DMEM/F12 and resuspend the solution by performing 5 up-and-down motions with a $10-\mathrm{ml}$ pipette.

p. Centrifuge for $10 \mathrm{~min}$ at $400 \times \mathrm{g}$.

q. Discard the supernatant. Use $4 \mathrm{ml} D M E M / F 12$ to resuspend the pellet. Subsequently, add $150 \mu$ dispase II at $0.5 \mathrm{mg} / \mathrm{ml}$ and incubate for $5 \mathrm{~min}$ at $37^{\circ} \mathrm{C}$.

r. Add $6 \mathrm{ml}$ DMEM/F12 and resuspend the solution by performing 5 up-and-down motions with a $10-\mathrm{ml}$ pipette.

s. Centrifuge for $10 \mathrm{~min}$ at $400 \times \mathrm{g}$.

t. Discard the supernatant. Resuspend the pellet in $9 \mathrm{ml}$ DMEM/F12.

u. Perform differential centrifugation to separate the mammary epithelium from the stromal fraction: centrifuge the suspension for $15 \mathrm{~s}$ at room temperature, $400 \times \mathrm{g}$. Discard the supernatant containing the stromal fraction and resuspend the epithelial pellet in $9 \mathrm{ml}$ $\mathrm{DMEM} / \mathrm{F} 12$. 
Note: Set the time on the centrifuge to $1 \mathrm{~min}$. Once a speed of $400 \times \mathrm{g}$ is reached, time 15 $s$ precisely and stop the centrifuge manually.

v. Repeat the previous step (t) 4 times, for a total of 5 differential centrifugations, to efficiently remove stromal contamination.

w. Resuspend the final pellet in $1 \mathrm{ml}$ basal organoid medium (BOM) and place on ice. The organoids are now ready to be counted and cultured.

Note: Adjust the volume of resuspension according to pellet size. From a pool of 2-3 mice, the final pellet was resuspended in $1 \mathrm{ml}$ basal organoid medium, for an expected range of 3,000-6,000 organoids. Adjust the volume of BOM for resuspension of the pellet according to the number of mice pooled. 
(A)

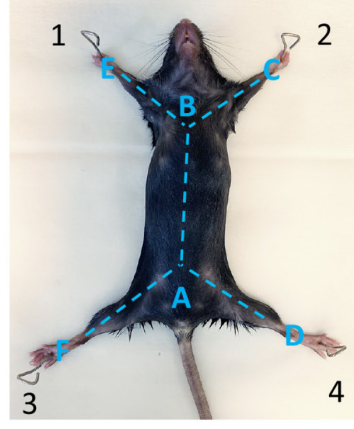

(B)
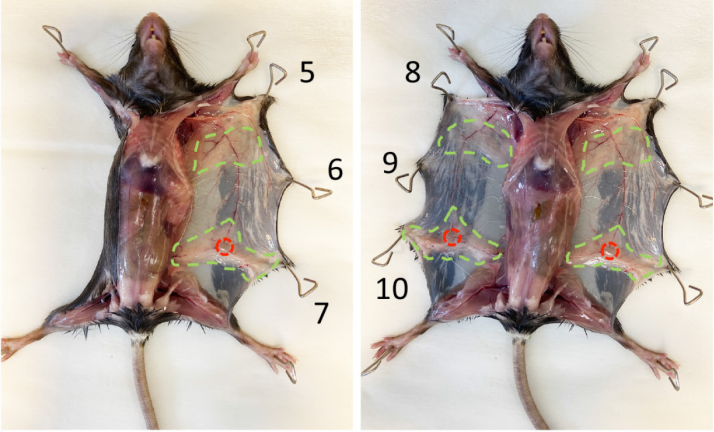

(C)

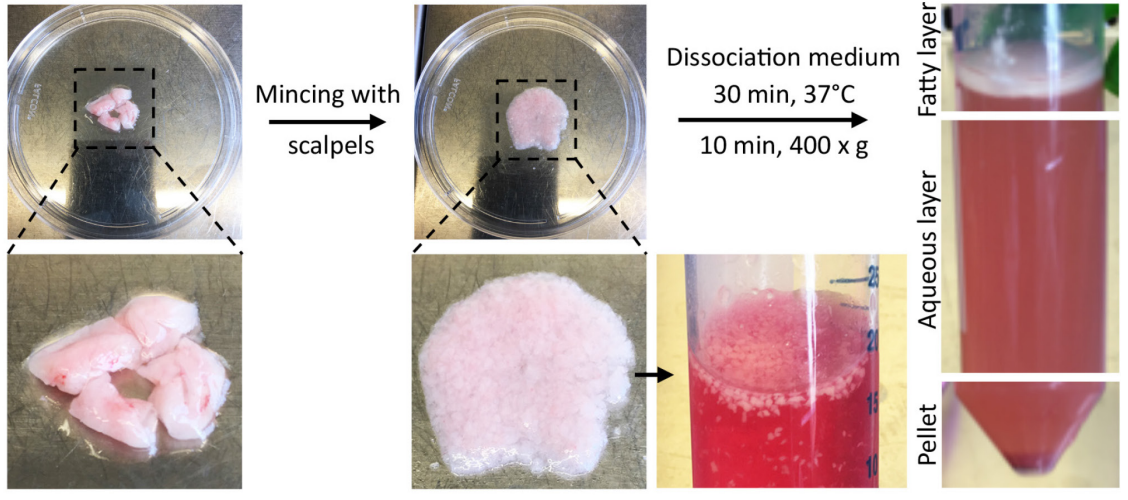

(D)

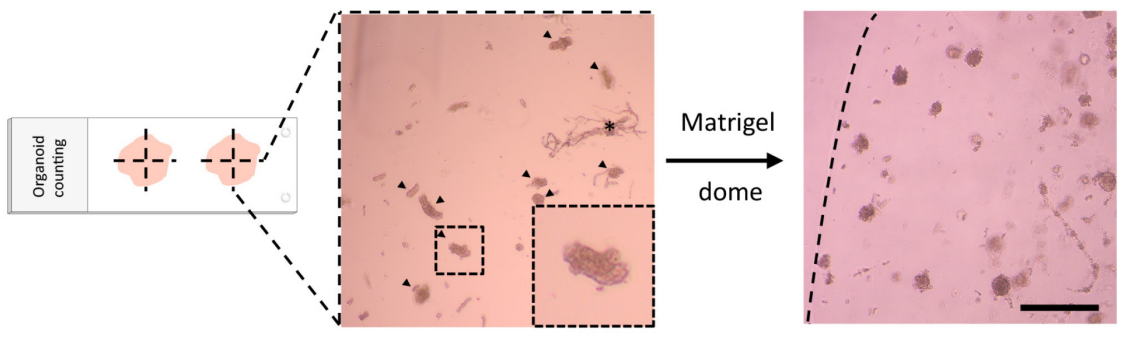

(E)

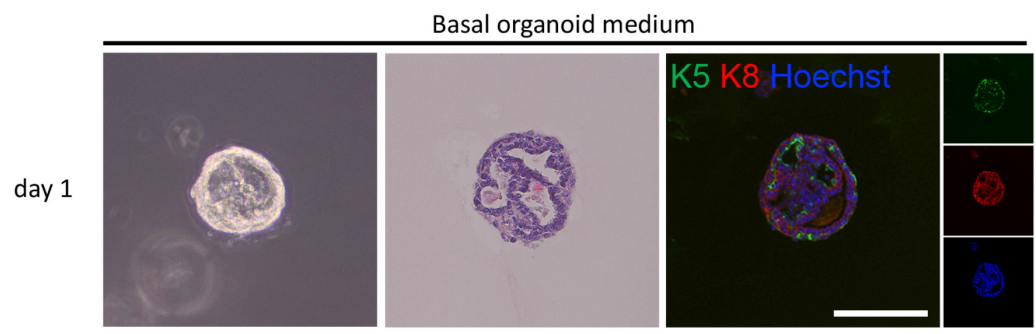

Figure 1. Key steps of mammary gland collection for organoid isolation and 3D culture.

A,B. Images of mouse dissection to access the mammary gland. A. Needles 1-4 represent the points at which to pin the mouse. Needles 5-7 and 8-10 represent the points at which to pin the skin of the mouse. Letters A-F with the blue dotted lines indicate the cuts. B. Green dotted lines denote the mammary gland. The lymph node is denoted in red and must be removed. C. Mammary gland before (left panel) and after (middle panel) mincing with a scalpel. Mammary organoids after transfer to dissociation medium (right panel). D. Example of mammary organoid counting. Left panel: organoids are surrounded by dotted lines. Star represents nerves. Right panel: organoids after embedding in Matrige ${ }^{\circledR}$. Arrow represents the edge of the Matrige ${ }^{\circledR}$ dome. Scale bar $=500 \mu \mathrm{m}$. E. Freshly isolated primary organoid. Left panel: image of a mammary 
organoid on day 1 post-isolation. Middle panel: Hematoxylin \& eosin staining of an organoid on day 1 post-isolation. Right panel: immunofluorescence staining showing the distribution of myoepithelial (keratin 5+, green) and luminal cells (keratin $8+$, red) in organoids on day 1 postisolation. Hoechst, blue (nuclei). Scale bar $=100 \mu \mathrm{m}$.

3. Organoid counting

Reminder: Work inside a laminar flow hood to maintain aseptic conditions.

a. Draw two large crosses with a marker on a microscope slide.

b. Take the organoid suspension and homogenize by performing five up-and-down motions with a P1000 pipette.

c. On the reverse side of the slide, spread $10 \mu$ solution around the center of each cross.

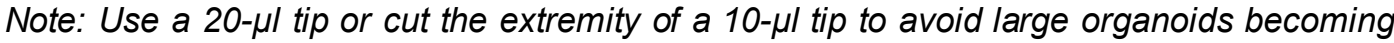
trapped.

d. Count the organoids under the microscope at $4 \times$ magnification (see Figure $1 \mathrm{D}$ ).

Notes:

i. Take each quarter of the cross as a landmark to avoid double-counting of the same organoid.

ii. Organoids appear as rounded structures with a smooth perimeter. Occasionally and unavoidably, nerves and endothelium are also present. The nerves appear as rope-like structures and can be organized in bundles (see Figure 1D). The endothelium has a somewhat ragged look in comparison with the smooth-looking organoids. The minor presence of primary nerves and endothelium does not interfere with organoid lactation or involution.

iii. Count only the organoids with a diameter greater than 30-50 $\mu \mathrm{m}$ since the smaller ones may not develop properly.

e. Calculate the average of the two counts in $10 \mu \mathrm{l}$ solution and multiply according to the volume of BOM used to resuspend the pellet to obtain the total number of organoids.

Note: Freshly isolated organoids can be viably frozen in a solution of FBS containing $10 \%$ DMSO for long-term storage in liquid nitrogen and later use.

B. 3D culture of mammary organoids

1. Embedding in Matrigel ${ }^{\circledR}$

Reminder: Work inside a laminar flow hood to maintain aseptic conditions. Wash the ice bucket and heating plate thoroughly with $70 \% \mathrm{EtOH}$ prior to placement in the laminar flow hood.

a. Thaw the Matrigel ${ }^{\circledR}$ on ice or at $4^{\circ} \mathrm{C}$.

Notes:

i. $\quad$ Matrige $^{\circledR}$ solidifies really fast at room temperature. Always keep it on ice before use and during the plating procedure. 
ii. Keep in mind that Matrige ${ }^{\circledR}$ thawing takes time; therefore, begin thawing prior to the procedure (2 $\mathrm{h}$ for a $1-\mathrm{ml}$ aliquot, $6 \mathrm{~h}$ for a $10-\mathrm{ml}$ bottle).

b. Place a 24-well plate on ice. Calculate the number of wells needed and spread 20- $\mu$ l Matrige $^{\circledR}$ in a round patch on the bottom of each well.

Note: Start by placing the tip containing Matrige ${ }^{\circledR}$ at the center of a well and expand circularly towards the edges of the well, without touching them.

c. Incubate the 24 -well plate in a cell incubator $\left(5 \% \mathrm{CO}_{2}\right)$ for $15 \mathrm{~min}$ at $37^{\circ} \mathrm{C}$.

d. In the meantime, pre-heat a heating plate to $37^{\circ} \mathrm{C}$.

e. Prepare the organoid suspension in the Matrige $^{\circledR}$ : calculate the volume of organoid suspension required to obtain the desired number of organoids. Pipette this volume of suspension into a fresh $1.5-\mathrm{ml}$ tube and centrifuge for $3 \mathrm{~min}$ at $400 \times \mathrm{g}$.

Note: Adjust the number of organoids per well depending on the type of experiment: 200 organoids per well for morphology and histology, 400 for gene expression, and 1000 for western blotting analysis.

f. Carefully remove the supernatant and place the tube on ice. Subsequently, carefully resuspend the pellet in the required volume of cold Matrige ${ }^{\circledR}(50 \mu \mathrm{l}$ per well), avoiding bubble formation. Keep on ice.

g. Remove the 24-well plate from the cell incubator and place on the $37^{\circ} \mathrm{C}$ heating plate.

h. In each Matrige ${ }^{\circledR}$-precoated well, cautiously seed the suspension of organoids (in Matrige ${ }^{\circledR}$ ) as a dome on top of the solidified Matrige ${ }^{\circledR}$ patch.

i. Place the 24-well plate back in the cell incubator $\left(5 \% \mathrm{CO}_{2}\right)$ for $30 \mathrm{~min}$ at $37^{\circ} \mathrm{C}$ to solidify the Matrige $^{\circledR}$ (see Figure 1D).

j. In the meantime, pre-warm $\mathrm{BOM}$ at $37^{\circ} \mathrm{C}$

k. Following incubation, carefully add $1 \mathrm{ml}$ pre-heated BOM to each well and culture in the cell incubator at $37^{\circ} \mathrm{C}, 5 \% \mathrm{CO}_{2}$.

Notes:

i. Add medium against the edges of the well to avoid disruption of the dome.

ii. Characterization of the organoids can be performed using regular histological stains (e.g., hematoxylin \& eosin) or immunostaining on day 1 post-recovery in BOM (see Figure $1 E$ and Step $B 2$ of the procedure).

2. Morphogenesis with FGF2

Reminder: Work inside a laminar flow hood to maintain aseptic conditions.

Note: Overnight recovery is optimal for organoid culture; however, FGF2 treatment can be administered immediately after plating the organoids.

a. Pre-heat the $\mathrm{BOM}$ at $37^{\circ} \mathrm{C}$.

b. Add fresh FGF2 at a final concentration of $2.5 \mathrm{nM}$ to pre-heated BOM to obtain the morphogenesis medium.

c. Aspirate the medium from the wells without touching the Matrige ${ }^{\circledR}$ dome and replace with $800 \mu$ fresh morphogenesis medium. 
d. Renew all medium with fresh morphogenesis medium every 3 days, for a total of 6 days of treatment.

3. Lactogenic differentiation with prolactin

a. Pre-heat the $B O M$ at $37^{\circ} \mathrm{C}$.

b. Add $1 \mu \mathrm{g} / \mathrm{ml}$ prolactin and $1 \mu \mathrm{g} / \mathrm{ml}$ hydrocortisone to the pre-heated BOM to obtain the lactation medium.

c. Aspirate the medium from the wells without touching the Matrige $\mathrm{l}^{\circledR}$ dome and replace with $800 \mu$ l fresh lactation medium.

d. Renew all medium with fresh lactation medium every two days, for a total of 4 days of treatment.

4. Myoepithelial cell contraction with oxytocin

a. Prepare fresh lactation medium, filter, and pre-heat at $37^{\circ} \mathrm{C}$.

b. Add $40 \mu \mathrm{g} / \mathrm{ml}$ recombinant oxytocin to the lactation medium.

c. Aspirate the medium from the wells without touching the Matrige ${ }^{\circledR}$ dome and replace with $800 \mu \mathrm{l}$ fresh lactation medium supplemented with oxytocin.

d. Using live cell imaging, record contraction images every second for $120 \mathrm{~s}$.

5. Mimicking involution by hormonal withdrawal

a. Pre-heat the $B O M$ at $37^{\circ} \mathrm{C}$.

b. Aspirate the medium from the wells without touching the Matrige ${ }^{\circledR}$ dome and replace with $800 \mu$ fresh BOM.

c. Renew all medium with BOM every two days, for a total of 8 days of treatment.

6. Replating

Note: Use tips pre-coated with $2.5 \%$ BSA.

a. Aspirate the supernatant and wash the wells twice with $800 \mu \mathrm{l}$ cold PBS.

b. Add $1 \mathrm{ml}$ cold PBS and disrupt the Matrige ${ }^{\circledR}$ dome using an up-and-down motion with a P1000 pipette.

c. Check for successful disintegration of the Matrige $\left.\right|^{\circledR}$ under a microscope.

d. Transfer the suspension to a 15-ml tube and add cold PBS to a total volume of $10 \mathrm{ml}$.

e. Centrifuge for $3 \mathrm{~min}$ at $400 \times \mathrm{g}$.

f. Carefully remove the supernatant, resuspend the organoid pellet in fresh Matrige ${ }^{\circledR}$ and plate as described in B1.

C. Organoid processing for further analysis

Note: We suggest carefully following organoid development under the microscope before renewing the media. Morphogenesis with FGF2 should induce branching after 3-4 days of treatment, while organoids in culture with BOM only, as the negative control, should remain round. Lactogenic differentiation can be confirmed by analysis of Csn2 mRNA using qPCR, comparing organoids before and after prolactin treatment ( $d 6$ versus d10). The involution process can also be confirmed using qPCR by detecting decreased expression of Csn2 mRNA following prolactin withdrawal (d10 
versus d18), or at the morphological level by the progressive disappearance of branching (see Figure $2 B$ and Figure $3 B$ ).

(A)

\begin{tabular}{|c|c|c|}
\hline + FGF2 & + PRL & \\
\hline & TTKL & Basal organoia meaium \\
\hline
\end{tabular}

(B)

$$
\text { Growing }
$$

(d6)

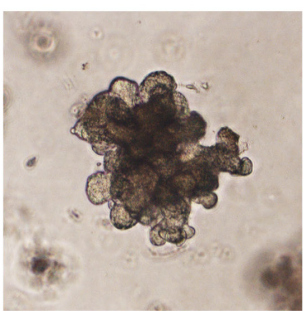

Lactation

(d10)

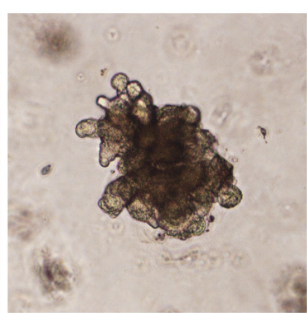

Involution

(d14)

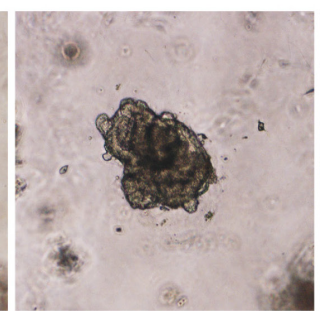

Involution

(d18)

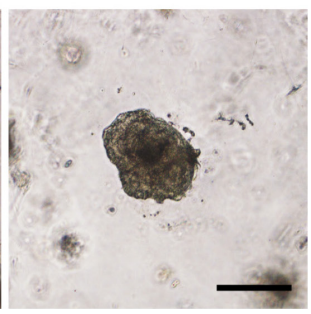

Figure 2. Modeling lactation and involution-like processes in primary mammary organoids. A. Scheme depicting the experimental design. B. Morphology of primary mammary organoids during lactation and involution-like processes. Bright-field images of organoid morphology following morphogenic and lactogenic stimulation and on days 4 or 8 after hormonal withdrawal. Scale bar $=100 \mu \mathrm{m}$.

(A)

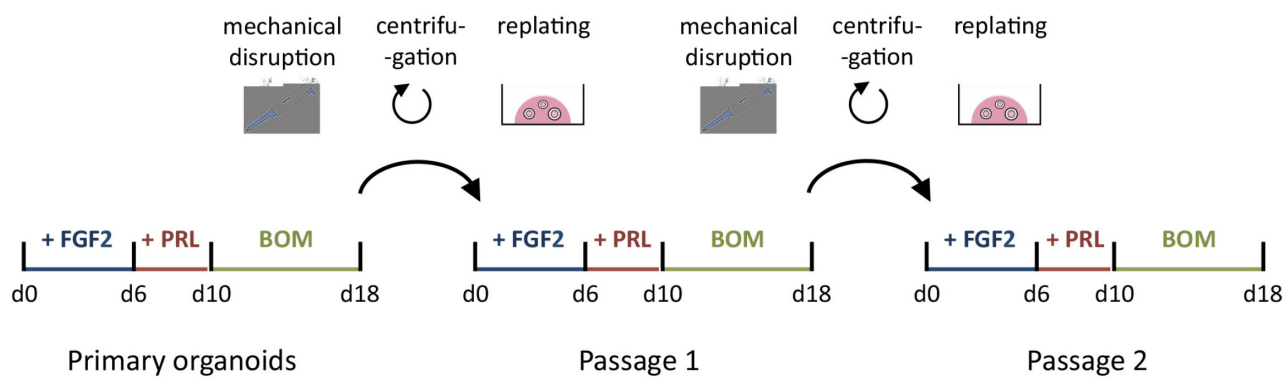

(B)

Growing

(d3)

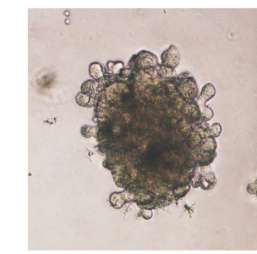

2

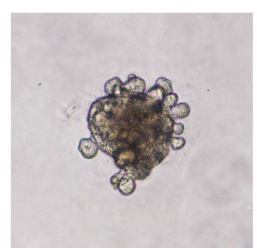

Growing

(d6)

\section{Lactation} (d10)
Involution

(d14)
Involution

(d18)
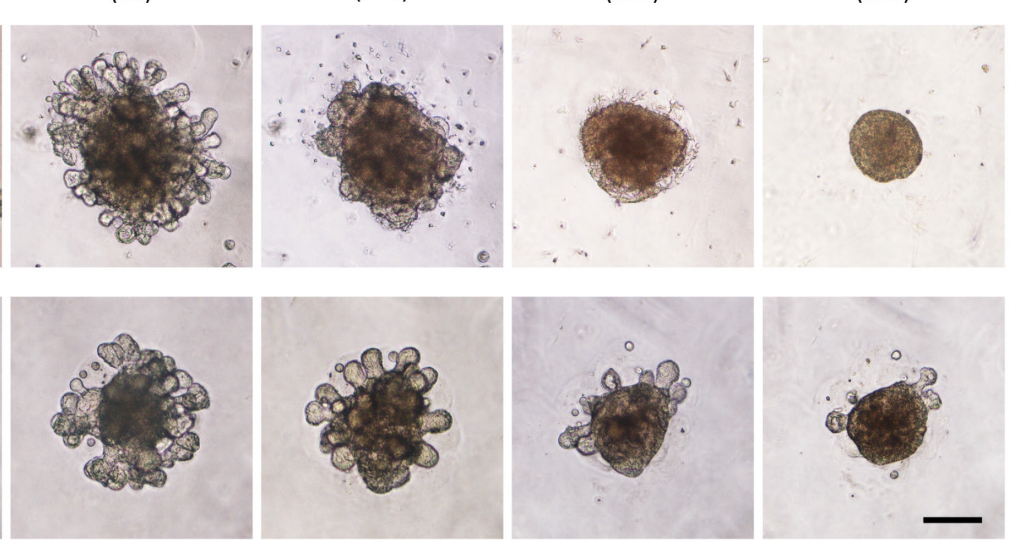
Figure 3. Passage of involution-like organoids. A. Scheme depicting the experimental design. PRL: Prolactin; BOM: basal organoid medium. B. Morphology of passaged organoids during the lactation and involution-like processes. Brightfield images of passage 1 (upper panel) and passage 2 (lower panel) organoids following morphogenic and lactogenic stimulation and on days 4 or 8 after hormonal withdrawal. Scale bar $=100 \mu \mathrm{m}$.

1. RNA isolation

Note: Embedding in Matrige ${ }^{\circledR}$ does not interfere with the quality of extracted RNA.

a. Aspirate the culture medium.

b. Add $350 \mu \mathrm{l}$ RLT buffer (RNeasy Micro Kit) contiaining $3.5 \mu \mathrm{l} \beta$-mercaptoethanol to each well.

c. Disintegrate the organoid culture in lysis buffer by performing ten up-and-down motions with a P1000 pipette.

d. Transfer the solution to a fresh 1.5-ml tube and vortex well.

Note: Samples can be stored at $-80^{\circ} \mathrm{C}$ until RNA extraction. To perform RNA extraction, thaw samples on ice and proceed according to the following instructions.

e. Homogenize RNA lysates by performing ten up-and-down motions with a single-use $30 \mathrm{G}$ insulin syringe.

f. Process samples as described in the RNeasy Micro Kit booklet, starting from Step C1b.

g. Measure the RNA concentration using a NanoDrop ${ }^{\mathrm{TM}}$.

2. Protein extraction

Note: Embedding in Matrige ${ }^{\circledR}$ interferes with western blotting analysis. Follow these steps to remove the Matrige ${ }^{\circledR}$ prior to protein extraction.

a. Aspirate the culture medium and dissociate the 3D culture with $800 \mu \mathrm{l}$ cold PBS supplemented with phosphatase inhibitor cocktail II.

b. Transfer the suspension to a clean 1.5-ml tube and centrifuge for $3 \mathrm{~min}$ at $400 \times \mathrm{g}, 4^{\circ} \mathrm{C}$.

c. Rinse twice with PBS supplemented with phosphatase inhibitor cocktail II.

d. Discard the supernatant and resuspend the pellet in $100 \mu$ ice-cold ready-to-use RIPA buffer supplemented with protease inhibitor cocktail I and phosphatase inhibitor cocktail II.

Note: Samples can be stored at $-80^{\circ} \mathrm{C}$ until protein extraction. To perform protein extraction, thaw samples on ice and proceed according to the following instructions.

e. Sonicate the samples twice at $4^{\circ} \mathrm{C}$ using a $60-\mathrm{kHz}$ ultrasonic wave frequency program (30 s ON/30 s OFF).

f. Vortex the samples, cool on ice, and repeat the sonication according to Step C2e.

g. Centrifuge for $20 \mathrm{~min}$ at $>10,000 \times \mathrm{g}, 4^{\circ} \mathrm{C}$.

h. Transfer the supernatant to a clean 1.5-ml tube.

i. Measure the protein concentration using a Coomassie Protein Assay Kit.

3. Fixation and embedding for histology

a. Aspirate the culture medium and rinse the culture twice with $800 \mu \mathrm{l}$ cold PBS. 
b. Incubate with $800 \mu \mathrm{l}$ 4\% PFA for 30 min. Following removal of the 4\% PFA, wash twice with PBS.

Notes:

i. Domes should be entirely covered with the solution. Add a greater volume if required.

ii. The fixed cultures can be stored in PBS at $4^{\circ} \mathrm{C}$ until embedding.

c. Prepare $3 \%$ low gelling temperature agarose in PBS and melt slowly in a microwave for $1.5-2 \mathrm{~min}$ at $1000 \mathrm{~W}$ (homogenize every $30 \mathrm{~s}$ by hand rotation).

d. Detach the fixed culture using the flat side of a spatula and transfer to a plastic histology mold containing melted agarose. Overlay with more agarose.

e. After solidification of the agarose, unmold the block. Use a scalpel to remove the excess agarose surrounding the Matrige ${ }^{\circledR}$ dome and place in a plastic embedding cassette for histology.

f. Proceed to sample dehydration: incubate the embedding cassettes in successive 1-h baths of $70 \% \mathrm{EtOH}, 95 \% \mathrm{EtOH}, 100 \% \mathrm{EtOH}$ (twice), xylene (twice), $50 \%$ xylene-50\% melted paraffin, and $100 \%$ melted paraffin.

g. Incubate overnight at $65^{\circ} \mathrm{C}$ in a second bath of $100 \%$ melted paraffin.

h. Embed in a histology tissue mold using an embedding workstation.

i. Unmold the paraffin blocks after $24 \mathrm{~h}$ of solidification.

j. Cut 5- $\mu \mathrm{m}$ sections and spread on microscope slides. Keep the slides at room temperature until further analysis.

k. Remove the paraffin prior to any staining by successive 5-min baths of xylene (twice), $100 \%$ EtOH (twice), 95\% EtOH, 70\% EtOH, and $\mathrm{H}_{2} \mathrm{O}$.

\section{Recipes}

1. Dissociation solution

Note: This solution is prepared inside a laminar flow hood under aseptic conditions and does not need to be filter-sterilized.

$2 \mathrm{mg} / \mathrm{ml}$ collagenase

$2 \mathrm{mg} / \mathrm{ml}$ trypsin

$5 \mu \mathrm{g} / \mathrm{ml}$ insulin

$50 \mu \mathrm{g} / \mathrm{ml}$ gentamicin

$5 \%$ FBS

$2 \mathrm{mM}$ glutamine

in DMEM/F12

2. BSA solution

Note: This solution can be filter-sterilized and reused several times when stored at $4^{\circ} \mathrm{C}$.

\section{$2.5 \%$ BSA in PBS}

3. Basal organoid medium (BOM) 
Note: This solution is prepared inside a laminar flow hood under aseptic conditions and does not need to be filter-sterilized.

$1 \times$ insulin-transferrin-selenium (ITS)

$100 \mathrm{U} / \mathrm{ml}$ penicillin

$100 \mu \mathrm{g} / \mathrm{ml}$ streptomycin

$2 \mathrm{mM}$ glutamine

in DMEM/F12

4. Morphogenesis medium

Note: This solution is prepared inside a laminar flow hood under aseptic conditions and does not need to be filter-sterilized.

\section{5 nM FGF2 in BOM}

5. Lactation medium

Note: This solution is prepared inside a laminar flow hood under aseptic conditions and does not need to be filter-sterilized.

$1 \mu \mathrm{g} / \mathrm{ml}$ prolactin

$1 \mu \mathrm{g} / \mathrm{ml}$ hydrocortisone

in BOM

6. $4 \%$ PFA

Note: This solution is prepared inside a chemical hood and does not need to be filter-sterilized. 4\% paraformaldehyde in PBS

7. RNA lysis buffer

Note: This solution is prepared inside a chemical hood and does not need to be filter-sterilized. $10 \mu \mathrm{l} \beta$-mercaptoethanol per $1 \mathrm{ml}$ RLT lysis buffer (from the RNeasy Micro Kit; this solution can be stored for up to one month at room temperature).

8. Phosphatase inhibitor cocktail II

Note: This solution is prepared inside a chemical hood or on a bench and does not need to be filter-sterilized.

$2 \mathrm{mM}$ imidazole

$1 \mathrm{mM}$ sodium fluoride

$1.15 \mathrm{mM}$ sodium molybdate

$1 \mathrm{mM}$ sodium orthovanadate

$4 \mathrm{mM}$ sodium tartrate dihydrate

in RIPA buffer

9. Protease inhibitor cocktail I

Note: This solution is prepared inside a chemical hood or on a bench and does not need to be filter-sterilized.

$500 \mu \mathrm{M}$ AEBSF hydrochloride

$150 \mathrm{nM}$ aprotinin

$1 \mu \mathrm{M}$ protease inhibitor E-64 


\author{
$0.5 \mathrm{mM}$ EDTA \\ $1 \mu \mathrm{M}$ leupeptin hemisulfate \\ in RIPA buffer
}

\title{
Acknowledgments
}

Work in the laboratory of $\mathrm{HL}$ is funded by the Pasteur, Centre National pour la Recherche the Agence Nationale de la Recherche (ANR-10-LABX-73 and ANR-16-CE13-0017- 01), Fondation ARC (PJA 20161205028 and 20181208231), Programme Barrande, and AFM-Telethon Foundation. AC was funded by postdoctoral fellowships from the Revive Consortium. EC was funded by a Ph.D. fellowship from Sorbonne Université. ZK was funded by the Grant Agency of Masaryk University (MUNI/G/1446/2018), Mobility grant by Ministry of Education, and Youth and Sports, and by funds from the Faculty of Medicine MU to the junior researcher (ROZV/28/LF/2020). JS was funded by the P-Pool (Faculty of Medicine MU) and the Grant Agency of Masaryk University (MUNI/A/1565/2018). This protocol was derived from the original research paper "Primary Mammary Organoid Model of Lactation and Involution" (Sumbal et al., 2020b).

\section{Competing interests}

The authors declare that they have no competing interests.

\section{Ethics}

The animal study was reviewed and approved by French legislation in compliance with European Communities Council Directives (A 75-15-01-3) and the regulations of the Institut Pasteur Animal Care Committees (CETEA).

\section{References}

1. Artegiani, B., and Clevers, H. (2018). Use and application of 3D-organoid technology. Hum Mol Genet 27: R99-R107.

2. Brisken, C. and O'Malley, B. (2010). Hormone action in the mammary gland. Cold Spring Harb Perspect Biol 2(12): a003178.

3. Brisken, C. and Rajaram, R. D. (2006). Alveolar and lactogenic differentiation. J Mammary Gland Biol Neoplasia 11(3-4): 239-248.

4. Campbell, J. J., Botos, L. A., Sargeant, T. J., Davidenko, N., Cameron, R. E. and Watson, C. J. (2014). A 3-D in vitro co-culture model of mammary gland involution. Integr Biol (Camb) 6(6): 618-626 
5. Ewald, A. J., Brenot, A., Duong, M., Chan, B. S. and Werb, Z. (2008). Collective epithelial migration and cell rearrangements drive mammary branching morphogenesis. Dev Cell 14(4): 570-581.

6. Freestone, D., Cater, M. A., Ackland, M. L., Paterson, D., Howard, D. L., de Jonge, M. D. and Michalczyk, A. (2014). Copper and lactational hormones influence the CTR1 copper transporter in PMC42-LA mammary epithelial cell culture models. J Nutr Biochem 25(4): 377-387.

7. Huch, M. and Koo, B. K. (2015). Modeling mouse and human development using organoid cultures. Development 142(18): 3113-3125.

8. Huebner, R. J., Neumann, N. M. and Ewald, A. J. (2016). Mammary epithelial tubes elongate through MAPK-dependent coordination of cell migration. Development 143: 983-993.

9. Hughes, K. and Watson, C. J. (2012). The spectrum of STAT functions in mammary gland development. JAKSTAT 1(3): 151-158.

10. Jamieson, P. R., Dekkers, J. F., Rios, A. C., Fu, N. Y., Lindeman, G. J. and Visvader, J. E. (2017). Derivation of a robust mouse mammary organoid system for studying tissue dynamics. Development 144(6): 1065-1071.

11. Jena, M. K., Jaswal, S., Kumar, S. and Mohanty, A. K. (2019). Molecular mechanism of mammary gland involution: An update. Dev Biol 445(2): 145-155.

12. Koledova, Z. (2017). 3D Cell Culture: An Introduction. Methods Mol Biol 1612: 1-11.

13. Linnemann, J. R., Miura, H., Meixner, L. K., Irmler, M., Kloos, U. J., Hirschi, B., Bartsch, H. S., Sass, S., Beckers, J., Theis, F. J., Gabka, C., Sotlar, K. and Scheel, C. H. (2015). Quantification of regenerative potential in primary human mammary epithelial cells. Development 142(18): 3239-3251.

14. Macias, H. and Hinck, L. (2012). Mammary gland development. Wiley Interdiscip Rev Dev Biol 1(4): 533-557.

15. Mroue, R., Inman, J., Mott, J., Budunova, I., and Bissell, M.J. (2015). Asymmetric expression of connexins between luminal epithelial- and myoepithelial- cells is essential for contractile function of the mammary gland. Dev Biol 399(1): 15-26.

16. Neumann, N. M., Perrone, M. C., Veldhuis, J. H., Huebner, R. J., Zhan, H., Devreotes, P. N., Brodland, G. W. and Ewald, A. J. (2018). Coordination of Receptor Tyrosine Kinase Signaling and Interfacial Tension Dynamics Drives Radial Intercalation and Tube Elongation. Dev Cell 45(1): 67-82 e66.

17. Ormandy, C. J., Camus, A., Barra, J., Damotte, D., Lucas, B., Buteau, H., Edery, M., Brousse, N., Babinet, C., Binart, N. and Kelly, P. A. (1997). Null mutation of the prolactin receptor gene produces multiple reproductive defects in the mouse. Genes Dev 11(2): 167-178.

18. Qu, Y., Han, B., Gao, B., Bose, S., Gong, Y., Wawrowsky, K., Giuliano, A. E., Sareen, D. and Cui, X. (2017). Differentiation of Human Induced Pluripotent Stem Cells to Mammary-like Organoids. Stem Cell Reports 8(2): 205-215.

19. Richert, M. M., Schwertfeger, K. L., Ryder, J. W. and Anderson, S. M. (2000). An atlas of mouse mammary gland development. J Mammary Gland Biol Neoplasia 5(2): 227-241. 
20. Shamir, E. R. and Ewald, A. J. (2015). Adhesion in mammary development: novel roles for Ecadherin in individual and collective cell migration. Curr Top Dev Biol 112: 353-382.

21. Sternlicht, M. D. (2006). Key stages in mammary gland development: the cues that regulate ductal branching morphogenesis. Breast Cancer Res 8(1): 201.

22. Sumbal, J., Budkova, Z., Traustadottir, G. A. and Koledova, Z. (2020a). Mammary Organoids and 3D Cell Cultures: Old Dogs with New Tricks. J Mammary Gland Biol Neoplasia. doi: 10.1007/s10911-020-09468-x

23. Sumbal, J., Chiche, A., Charifou, E., Koledova, Z. and Li, H. (2020b). Primary Mammary Organoid Model of Lactation and Involution. Front Cell Dev Biol 8: 68.

24. Xian, W., Schwertfeger, K. L., Vargo-Gogola, T. and Rosen, J. M. (2005). Pleiotropic effects of FGFR1 on cell proliferation, survival, and migration in a 3D mammary epithelial cell model. $J$ Cell Biol 171(4): 663-673.

25. Zwick, R. K., Rudolph, M. C., Shook, B. A., Holtrup, B., Roth, E., Lei, V., Van Keymeulen, A., Seewaldt, V., Kwei, S., Wysolmerski, J., Rodeheffer, M. S. and Horsley, V. (2018). Adipocyte hypertrophy and lipid dynamics underlie mammary gland remodeling after lactation. Nat Commun 9(1): 3592. 